\title{
An Existence Result for Discontinuous
}

\section{Games *}

\author{
Guilherme Carmona ${ }^{\mathrm{a}, *}$, \\ ${ }^{a}$ Universidade Nova de Lisboa
}




\section{Abstract}

We introduce a notion of upper semicontinuity, weak upper semicontinuity, and show that it, together with a weak form of payoff security, is enough to guarantee the existence of Nash equilibria in compact, quasiconcave normal form games. We show that our result generalizes the pure strategy existence theorem of Dasgupta and Maskin [3] and that it is neither implied nor does it imply the existence theorems of Baye, Tian, and Zhou [2] and Reny [5]. Furthermore, we show that an equilibrium may fail to exist when, while maintaining weak payoff security, weak upper semicontinuity is weakened to reciprocal upper semicontinuity.

Journal of Economic Literature Classification Numbers: C72

Key words: Discontinuous Games; Nash Equilibrium; Upper Semicontinuity;

Lower Semicontinuity

\footnotetext{
^ I wish to thank Iliyan Georgiev for very helpful comments. I thank also John Huffstot for editorial assistance. Any remaining errors are, of course, mine.

* Corresponding author: Universidade Nova de Lisboa, Faculdade de Economia, Campus de Campolide, 1099-032 Lisboa, Portugal.

Email address: gcarmona@fe.unl.pt (Guilherme Carmona).
} 


\section{Introduction}

The existence of Nash equilibria in compact, quasiconcave games relies on some weak form of upper and lower semicontinuity. These two semicontinuity requirements can be combined in one single condition, such as better-reply security ([5, Theorem 3.1$])$ or be imposed separately as weak reciprocal upper semicontinuity and payoff security, respectively ([1, Proposition 1]). Also, as in [3, Corollary], they can take the form of upper semicontinuity (i.e., each player's payoff function is upper semicontinuous) and weak payoff security (i.e., each player's value function is lower semicontinuous).

Comparing the last two sets of conditions suggests that weakening the lower semicontinuity requirement from payoff security to weak payoff security requires a strengthening of the upper semicontinuity one. We show that this is the case by presenting an example of a game that, while satisfying weak reciprocal upper semicontinuity and weak payoff security, fails to have a Nash equilibrium. In fact, the same conclusion holds even if we require the sum of players' payoff functions to be smooth.

Furthermore, we show that a considerably weaker condition than upper semicontinuity suffices for the existence of Nash equilibria in weakly payoff-secure games. This condition, which we name weak upper semicontinuity, strengthens the notion of weak reciprocal upper semicontinuity of Bagh and Jofre [1]. This is accomplished by imposing a similar requirement as theirs, although not on the players' payoff function but rather on an extended payoff function that allows each player to deviate singly. ${ }^{1}$

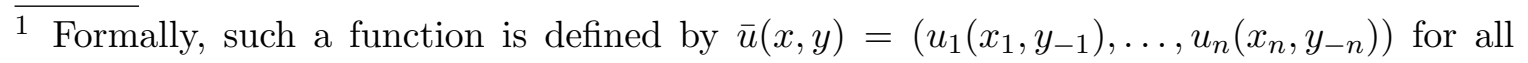


Our existence result then states that a compact, quasiconcave game has a Nash equilibrium provided that it is weakly upper semicontinuous and weakly payoff secure.

The above existence result is useful for games whose critical discontinuities are of the lower, but not the upper, semicontinuity type. Indeed, we present examples of games that satisfy the assumptions of our existence result but fail to have enough lower semicontinuity to allow us to use either Theorem 3.1 in [5] or Theorem 1 in [2]. Similarly, we also present examples of games that satisfy the assumptions of the existence results of Reny and Baye, Tian and Zhou, but fail to have enough upper semicontinuity to allow us to use our existence result.

Our existence result is obtained through a characterization of games that satisfy both weak upper semicontinuity and weak payoff security. We show that a game satisfies these conditions if and only if each player's value function is continuous and limit points of a player's approximate best-replies, with the level of approximation converging to zero, are themselves best-replies. This characterization clearly implies that the (joint) best-reply correspondence has nonempty and compact values and a closed graph, from which the existence of a Nash equilibrium can be derived using standard arguments.

Although weak upper semicontinuity is a relatively weak condition, our characterization also reveals that it becomes quite demanding when combined with weak payoff security. In fact, it roughly requires that, when a player's payoff function jumps down

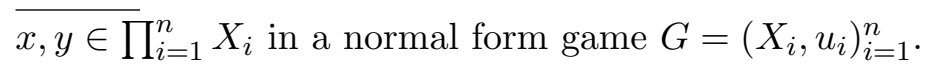


at a strategy $x$, that player has a strategy yielding a payoff strictly higher than those he would obtain in a neighborhood of $x$. However, unlike upper semicontinuity, weak upper semicontinuity allows a player's payoff function to jump down provided that that player can compensate such a fall somewhere in his action space. This weakening of upper semicontinuity is analogous to that of reciprocal upper semicontinuity obtained through weak reciprocal upper semicontinuity, since the latter allows all players' payoffs to jump down provided that some player can compensate such a fall somewhere in his action space. Thus, our existence result is better regarded as a generalization of the existence result of Dasgupta and Maskin [3] in the same spirit of the generalization of Reny's Corollary 3.3 obtained by Bagh and Jofre [1].

\section{Notation and Definitions}

A normal form game $G$ consists of a finite set of players $N=\{1, \ldots, n\}$ and, for all players $i \in N$, a metric space $X_{i}$ of pure strategies and a payoff function $u_{i}: X \rightarrow \mathbb{R}$, where $X=\prod_{i \in N} X_{i}$.

We say that a game $G$ is compact if, for all $i \in N, X_{i}$ is compact and $u_{i}$ is bounded (equivalently, $u_{i}(X)$ is contained in a compact subset of $\mathbb{R}$ ). Given a player $i \in N$, the symbol $-i$ denotes "all players but $i$ ". Also, $X_{-i}=\prod_{j \neq i} X_{j}$. Furthermore, we say that $G$ is quasiconcave if, for all $i \in N, X_{i}$ is convex and $u_{i}\left(\cdot, x_{-i}\right)$ is quasiconcave for all $x_{-i} \in X_{-i}$.

Let, for all $i \in N, v_{i}: X_{-i} \rightarrow \mathbb{R}$ be defined by $v_{i}\left(x_{-i}\right)=\sup _{x_{i} \in X_{i}} u_{i}\left(x_{i}, x_{-i}\right)$; the 
function $v_{i}$ is player $i$ 's value function. A game $G$ is weakly payoff secure if $v_{i}$ is lower semicontinuous for all $i \in N$. This assumption was introduced in [3] and is easily seen to be equivalent to the following weak form of payoff security (hence its name): for all $i \in N, x \in X$ and $\varepsilon>0$, there exists $\delta>0$ with the following property: for all $x_{-i}^{\prime} \in B_{\delta}\left(x_{-i}\right)$ there exists $\bar{x}_{i} \in X_{i}$ such that $u_{i}\left(\bar{x}_{i}, x_{-i}^{\prime}\right) \geq u_{i}\left(x_{i}, x_{-i}\right)-\varepsilon$.

A game $G$ is upper semicontinuous if $u_{i}$ is upper semicontinuous for all $i \in N$. A weaker notion of upper semicontinuity is defined as follows. Define $\bar{u}: X \times X \rightarrow$ $\mathbb{R}^{n}$ by $\bar{u}(x, y)=\left(u_{1}\left(x_{1}, y_{-1}\right), \ldots, u_{n}\left(x_{n}, y_{-n}\right)\right)$ and let $\Gamma=\operatorname{cl}(\operatorname{graph}(\bar{u})) \backslash \operatorname{graph}(\bar{u})$ denote the frontier of the graph of $\bar{u} .^{2}$ A game $G$ is weakly upper semicontinuous if for all $(x, y, u) \in \Gamma$ there exists $i \in N$ and $\hat{x}_{i} \in X_{i}$ such that $u_{i}\left(\hat{x}_{i}, y_{-i}\right)>u_{i}$. Proposition 3 below shows that, indeed, if $G$ is upper semicontinuous, then $G$ is weakly reciprocally semicontinuous. This notion is inspired by and strengthens the notion of weak reciprocal upper semicontinuity introduced by [1]. The function $\bar{u}$ is used in [5] and also in [2] under the form $\sum_{i \in N} \bar{u}_{i}$.

\section{Existence of Nash Equilibria}

In this section, we characterize games that satisfy both weak upper semicontinuity and weak payoff security and use such characterization to derive our existence result (Subsection 3.1). In Subsection 3.2, we show that our existence result strictly generalizes the existence result of [3] and that it is formally unrelated to those of [5] and

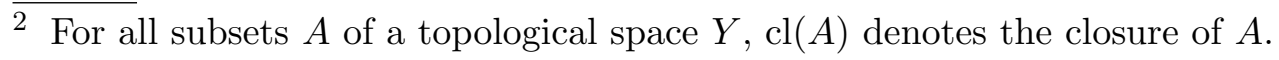


[2]. ${ }^{3}$ Finally, in Subsection 3.3, we present an example showing that reciprocal upper semicontinuity together with weak payoff security are not enough to guarantee the existence of Nash equilibria.

\subsection{An Existence Result}

The following result provides a characterization of weakly payoff secure and weakly upper semicontinuous games.

Theorem 1 Let $G$ be a compact game. Then, the following conditions are equivalent:

(1) $G$ is weakly payoff secure and weakly upper semicontinuous.

(2) $G$ is weakly payoff secure and for all $i \in N$ and $(x, \alpha) \in \operatorname{cl}\left(\operatorname{graph}\left(u_{i}\right)\right) \backslash \operatorname{graph}\left(u_{i}\right)$, there exists $\hat{x}_{i} \in X_{i}$ such that $u_{i}\left(\hat{x}_{i}, x_{-i}\right)>\alpha$.

(3) For all $i \in N, v_{i}$ is continuous and the following property holds: if $x \in X$, $\left\{x_{k}\right\}_{k=1}^{\infty} \subseteq X$ and $\left\{\varepsilon_{k}\right\}_{k=1}^{\infty} \subseteq \mathbb{R}_{+}$are such that $\lim _{k} x_{k}=x, \lim _{k} \varepsilon_{k}=0$ and $u_{i}\left(x_{k}\right) \geq v_{i}\left(x_{-i}^{k}\right)-\varepsilon_{k}$ for all $k \in \mathbb{N}$, then $u_{i}(x)=v_{i}\left(x_{-i}\right)$.

The equivalence between the first two conditions shows that, when combined with weak payoff security, the requirement of weak upper semicontinuity becomes quite demanding. An alternative way of seeing this is by defining a function $\hat{u}: X^{n} \rightarrow \mathbb{R}^{n}$ by $\hat{u}\left(x^{1}, \ldots, x^{n}\right)=\left(u_{1}\left(x^{1}\right), \ldots, u_{n}\left(x^{n}\right)\right)$ and noting that the second condition in 2 holds if and only if for all $\left(x^{1}, \ldots, x^{n}, u\right) \in \operatorname{cl}(\operatorname{graph}(\hat{u})) \backslash \operatorname{graph}(\hat{u})$, there exists $i \in N$ $\overline{3}$ Note that Theorem 2 of [3] is strictly weaker than their corollary. See the working paper version of this paper for details. 
and $\hat{x}_{i} \in X_{i}$ such that $u_{i}\left(\hat{x}_{i}, x_{-i}^{i}\right)>u_{i}$. Thus, the difference between weak reciprocal upper semicontinuity, weak upper semicontinuity imposed alone and weak reciprocal upper semicontinuity combined with weak payoff security is due to how the limit payoff vector is computed: for weak reciprocal upper semicontinuity, this vector is computed requiring all players to use the same strategy, whereas for weak upper semicontinuity this requirement is relaxed by allowing each player to change her own strategy; furthermore, when weak upper semicontinuity is combined with weak payoff security, the limit payoff vector is computed allowing different players to use different strategies.

The equivalence between the first and third conditions implies that, in compact, weakly upper semicontinuous, weakly payoff-secure games, the best-reply correspondence has nonempty and compact values and a closed graph. Furthermore, it also has convex values whenever the game is quasiconcave. In this case, it follows from [4] that the best-reply correspondence has a fixed point, and so the game has a Nash equilibrium. In summary, Theorem 1 implies the following existence result. ${ }^{4}$

Corollary 2 If $G$ is compact, quasiconcave, weakly upper semicontinuous and weakly payoff secure, then $G$ has a Nash equilibrium.

We conclude this subsection with the proof of Theorem 1.

4 The equivalence between the first and third condition shows that, besides the continuity of players' value functions, weak upper semicontinuity and weak payoff security are almost necessary for the best-reply property to have the standard fixed point theorem's assumptions. 
Proof of Theorem 1. We start by establishing that 1 implies 2 . Let $i \in N$ and $(x, \alpha) \in \operatorname{cl}\left(\operatorname{graph}\left(u_{i}\right)\right) \backslash \operatorname{graph}\left(u_{i}\right)$. If $u_{i}(x)>\alpha$, simply let $\hat{x}_{i}=x_{i}$; thus, we may assume that $u_{i}(x)<\alpha$. Let $\left\{x_{k}\right\}_{k=1}^{\infty}$ be such that $\lim _{k} x_{k}=x$ and $\lim _{k} u_{i}\left(x_{k}\right)=\alpha$. For all $k \in \mathbb{N}$, define $z_{i}^{k}=x_{i}^{k}$ and, for all $j \neq i$, let $z_{j}^{k} \in X_{j}$ be such that $u_{j}\left(z_{j}^{k}, x_{-j}^{k}\right)>$ $v_{j}\left(x_{-j}^{k}\right)-1 / k$. Since $G$ is compact, we may assume that $\left\{z_{k}\right\}_{k=1}^{\infty}$ and $\left\{v_{j}\left(x_{-j}^{k}\right)\right\}_{k=1}^{\infty}$ converge for all $j \neq i$. Let $z=\lim _{k} z_{k}$ and $\alpha_{j}=\lim _{k} v_{j}\left(x_{-j}^{k}\right)$ for all $j \neq i$. Since $G$ is weakly payoff secure, then $v_{j}\left(x_{-j}\right) \leq \alpha_{j}$ and so $u_{j}\left(\hat{x}_{j}, x_{-j}\right) \leq \alpha_{j}$ for all $j \neq i$ and $\hat{x}_{j} \in X_{j}$. Hence, by weak upper semicontinuity, there exists $\hat{x}_{i} \in X_{i}$ such that $u_{i}\left(\hat{x}_{i}, x_{-i}\right)>\alpha$

We show next that 2 implies 3 . Let $i \in N, x_{-i} \in X_{-i}$ and $\left\{x_{-i}^{k}\right\}_{k=1}^{\infty}$ be such that $\lim _{k} x_{-i}^{k}=x_{-i}$. Let $\left\{v_{i}\left(x_{-i}^{m}\right)\right\}_{m=1}^{\infty}$ be a subsequence of $\left\{v_{i}\left(x_{-i}^{k}\right)\right\}_{k=1}^{\infty}$. For all $m \in \mathbb{N}$, let $x_{i}^{m} \in X_{i}$ be such that $u_{i}\left(x_{i}^{m}, x_{-i}^{m}\right)>v_{i}\left(x_{-i}^{m}\right)-1 / m$. Since $G$ is compact, then there is a subsequence $\left\{v_{i}\left(x_{-i}^{n}\right)\right\}_{n=1}^{\infty}$ of $\left\{v_{i}\left(x_{-i}^{m}\right)\right\}_{m=1}^{\infty}$ and a subsequence $\left\{x_{i}^{n}\right\}_{n=1}^{\infty}$ of $\left\{x_{i}^{m}\right\}_{m=1}^{\infty}$ such that both converge. By construction, $\lim _{n} u_{i}\left(x_{n}\right)=\lim _{n} v_{i}\left(x_{-i}^{n}\right)$ and so $\left(x, \lim _{n} v_{i}\left(x_{-i}^{n}\right)\right) \in \operatorname{cl}\left(\operatorname{graph}\left(u_{i}\right)\right)$. Since $u_{i}(x) \leq v_{i}\left(x_{-i}\right) \leq \lim _{n} v_{i}\left(x_{-i}^{n}\right)$, it follows by 2 that $u_{i}(x)=\lim _{n} v_{i}\left(x_{-i}^{n}\right)$ and so $v_{i}\left(x_{-i}\right)=\lim _{n} v_{i}\left(x_{-i}^{n}\right)$. Since every subsequence of $\left\{v_{i}\left(x_{-i}^{k}\right)\right\}_{k=1}^{\infty}$ has a further subsequence converging to $v_{i}\left(x_{-i}\right)$, we conclude that $v_{i}\left(x_{-i}\right)=\lim _{k} v_{i}\left(x_{-i}^{k}\right)$ and that $v_{i}$ is continuous.

In order to establish the second condition in 3 , consider $x \in X,\left\{x_{k}\right\}_{k=1}^{\infty} \subseteq X$ and $\left\{\varepsilon_{k}\right\}_{k=1}^{\infty} \subseteq \mathbb{R}_{+}$such that $\lim _{k} x_{k}=x, \lim _{k} \varepsilon_{k}=0$ and $u_{i}\left(x_{k}\right) \geq v_{i}\left(x_{-i}^{k}\right)-\varepsilon_{k}$ for all $k \in \mathbb{N}$. Then, $\lim _{k} u_{i}\left(x_{k}\right)=\lim _{k} v_{i}\left(x_{-i}^{k}\right)=v_{i}\left(x_{-i}\right)$. Thus, $\left(x, v_{i}\left(x_{-i}\right)\right) \in \operatorname{cl}\left(\operatorname{graph}\left(u_{i}\right)\right)$ and so $u_{i}(x)=v_{i}\left(x_{-i}\right)$. 
We finally establish that 3 implies 1 . Since $v_{i}$ is continuous for all $i \in N$, then $G$ is weakly payoff secure. In order to see that $G$ is weakly upper semicontinuous, consider $(x, y, u) \in \Gamma$ and let $\left\{\left(x_{k}, y_{k}\right)\right\}_{k=1}^{\infty}$ be such that $(x, y)=\lim _{k}\left(x_{k}, y_{k}\right)$ and $u=$ $\lim _{k} \bar{u}\left(x_{k}, y_{k}\right)$. If $u_{i}=v_{i}\left(y_{-i}\right)$ for all $i \in N$, then, letting $\varepsilon_{i}^{k}=v_{i}\left(y_{-i}^{k}\right)-u_{i}\left(x_{i}^{k}, y_{-i}^{k}\right) \geq 0$ and $\varepsilon_{k}=\max _{i} \varepsilon_{k}^{i}$ for all $k \in \mathbb{N}$ and $i \in N$, we obtain $u_{i}\left(x_{i}^{k}, y_{-i}^{k}\right) \geq v_{i}\left(y_{-i}^{k}\right)-\varepsilon_{k}$, $\lim _{k} \varepsilon_{k}^{i}=v_{i}\left(y_{-i}\right)-u_{i}=0$ for all $i \in N$ and so $\lim _{k} \varepsilon_{k}=0$. It then follows by 3 that $\bar{u}_{i}(x, y)=u_{i}\left(x_{i}, y_{-i}\right)=v_{i}\left(y_{-i}\right)=u_{i}$ and so $(x, y, u) \in \operatorname{graph}(\bar{u})$, a contradiction. Hence, there exists $i \in N$ such that $u_{i} \neq v_{i}\left(y_{-i}\right)$. Since $u_{i}=\lim _{k} u_{i}\left(x_{i}^{k}, y_{-i}^{k}\right) \leq$ $\lim _{k} v_{i}\left(y_{-i}^{k}\right)=v_{i}\left(y_{-i}\right)$, then $u_{i}<v_{i}\left(y_{-i}\right)$ and so there exists $\hat{x}_{i} \in X_{i}$ such that $u_{i}\left(\hat{x}_{i}, y_{-i}\right)>u_{i}$. Therefore, $G$ is weakly upper semicontinuous.

\subsection{Examples and Related Literature}

Most of the examples we consider belong to the following class of diagonal games. These are two-player games on the unit square with the following payoff functions:

$$
u_{i}(x)=\left\{\begin{array}{lll}
\phi_{i}(x) & \text { if } & x_{i}=x_{-i} \\
f_{i}(x) & \text { if } & x_{i} \neq x_{-i}
\end{array}\right.
$$

where $\phi_{i}, f_{i}:[0,1]^{2} \rightarrow \mathbb{R}$ are continuous.

Clearly, a diagonal game $G$ is compact. Suppose, in addition, that $G$ is also quasiconcave and satisfies the following condition: for all $i \in\{1,2\}$ and $x_{-i} \in[0,1]$, either $\phi_{i}\left(x_{-i}, x_{-i}\right)=v_{i}\left(x_{-i}\right)$ or there exists $\hat{x}_{i} \in[0,1]$ such that $f_{i}\left(\hat{x}_{i}, x_{-i}\right)>f_{i}\left(x_{-i}, x_{-i}\right)$. 
When a diagonal game satisfies these two assumptions, one can show that it satisfies the conditions of Corollary 2 and so that it has a Nash equilibrium.

In fact, weak payoff security follows because

$$
v_{i}\left(x_{-i}\right)=\max \left\{\phi_{i}\left(x_{-i}, x_{-i}\right), \max _{x_{i} \in[0,1]} f_{i}\left(x_{i}, x_{-i}\right)\right\}
$$

for all $i \in\{1,2\}$ and $x_{-i} \in[0,1]$ and so $v_{i}$ is continuous. Note that the above equality clearly holds when $\phi_{i}\left(x_{-i}, x_{-i}\right) \geq \max _{x_{i} \in[0,1]} f_{i}\left(x_{i}, x_{-i}\right)$. When $\phi_{i}\left(x_{-i}, x_{-i}\right)<$ $\max _{x_{i} \in[0,1]} f_{i}\left(x_{i}, x_{-i}\right)$, then $\max _{x_{i} \in[0,1]} f_{i}\left(x_{i}, x_{-i}\right)>f_{i}\left(x_{-i}, x_{-i}\right)$. This conclusion follows since otherwise we obtain that $\phi_{i}\left(x_{-i}, x_{-i}\right)<f_{i}\left(x_{-i}, x_{-i}\right)$ and so $\phi_{i}\left(x_{-i}, x_{-i}\right)<$ $f_{i}\left(x_{i}^{\prime}, x_{-i}\right)$ for some $x_{i}^{\prime}$ sufficiently close to $x_{-i}$. Hence, $v_{i}\left(x_{-i}\right)>\phi_{i}\left(x_{-i}, x_{-i}\right)$ and so $f_{i}\left(\hat{x}_{i}, x_{-i}\right)>f_{i}\left(x_{-i}, x_{-i}\right)=\max _{x_{i}} f_{i}\left(x_{i}, x_{-i}\right)$ for some $\hat{x}_{i} \neq x_{-i}$, a contradiction. Clearly, $\max _{x_{i} \in[0,1]} f_{i}\left(x_{i}, x_{-i}\right)>\max \left\{f_{i}\left(x_{-i}, x_{-i}\right), \phi_{i}\left(x_{-i}, x_{-i}\right)\right\}$ implies that $v_{i}\left(x_{-i}\right)=$ $\max _{x_{i} \in[0,1]} f_{i}\left(x_{i}, x_{-i}\right)$.

In order to establish the weak upper semicontinuity of $G$, let $(x, y, u) \in \Gamma$ and consider a sequence $\left\{\left(x_{k}, y_{k}\right)\right\}_{k=1}^{\infty}$ converging to $(x, y)$ such that $\left\{\bar{u}\left(x_{k}, y_{k}\right)\right\}_{k=1}^{\infty}$ converges to $u$. We may assume that $u_{i}\left(x_{i}, y_{-i}\right)<u_{i}$ for some $i$, since otherwise there is nothing to prove. It is clear that $u_{i}=u_{i}\left(x_{i}, y_{-i}\right)$ if either $x_{i} \neq y_{-i}$, or if $x_{i}=y_{-i}$ and there exists a subsequence $\left\{\left(x_{i}^{m}, y_{-i}^{m}\right)\right\}_{m=1}^{\infty}$ of $\left\{\left(x_{i}^{k}, y_{-i}^{k}\right)\right\}_{k=1}^{\infty}$ such that $x_{i}^{m}=y_{-i}^{m}$ for all $j$. Hence, $x_{i}=$ $y_{-i}$ and $x_{i}^{k} \neq y_{-i}^{k}$ for all $k$ sufficiently large. Therefore, $f_{i}\left(y_{-i}, y_{-i}\right)=u_{i}>u_{i}\left(x_{i}, y_{-i}\right)=$ $\phi_{i}\left(y_{-i}, y_{-i}\right)$. The continuity of $f_{i}$ implies that $v_{i}\left(y_{-i}\right) \geq f_{i}\left(x_{i}^{\prime}, y_{-i}\right)>\phi_{i}\left(y_{-i}, y_{-i}\right)$ for some $x_{i}^{\prime} \neq y_{-i}$ and so there exists $\hat{x}_{i} \neq y_{-i}$ such that $u_{i}\left(\hat{x}_{i}, y_{-i}\right)>f_{i}\left(y_{-i}, y_{-i}\right)=u_{i}$, establishing the desired conclusion. 
While a quasiconcave, diagonal game satisfying the above conditions always satisfies the assumptions of Corollary 2, it may fail to satisfy better-reply security. When this is the case, Reny's theorem cannot be applied.

The following example illustrates that a quasiconcave, diagonal game may fail to be better-reply secure. Consider two friends who have to catch a train at 9am and want to meet sometime between 8 and 9am. While one of them is very strict on punctuality, the other likes to be 5 minutes late. Formally, let $[0,1]$ represent a time between 8 and 9am, $\varepsilon>0$ represent a delay of 5 minutes and player 1 represent the punctual friend. Assume that $G$ is quasiconcave, $\phi_{1}(x)>0$ for all $x \in[0,1]^{2}, f_{1} \equiv 0$, $u_{2}\left(x_{1}, x_{1}+\varepsilon\right)>u_{2}\left(x_{1}, x_{2}\right)$ for all $x_{1}<1-\varepsilon$ and $x_{2} \neq x_{1}+\varepsilon$ and $u_{2}\left(x_{1}, 1\right)>u_{2}\left(x_{1}, x_{2}\right)$ for all $x_{1} \geq 1-\varepsilon$ and $x_{2} \neq 1 .^{5}$ Then, it follows from Theorem 2 and the above analysis that this game as a Nash equilibrium. ${ }^{6}$ In fact, this game has a unique Nash equilibrium $x_{1}=x_{2}=1$, i.e., players meet only when the train is about to leave. However, this game is not better-reply secure. To see this, simply consider $x=(0, \varepsilon)$ and $u=\left(0, f_{2}(0, \varepsilon)\right)$. Clearly, $(x, u) \in \operatorname{graph}(u), x$ is not a Nash equilibrium, player 2 cannot obtain a payoff strictly above $u_{2}$, and player 1 , while able to obtain a payoff strictly above 0, cannot secure it. Hence, Reny's Theorem cannot be applied to this game.

$\overline{5}$ For example, let $u_{2}(x)=\left(1-x_{1}+x_{2}\right) /(1+\varepsilon)$ if $x_{2} \leq x_{1}+\varepsilon$ and $u_{2}(x)=\left(1+2 \varepsilon+x_{1}-\right.$ $\left.x_{2}\right) /(1+\varepsilon)$ otherwise.

6 The quasiconcavity of $u_{2}\left(x_{1}, \cdot\right)$ implies that $\max _{x_{2}} f_{2}\left(x_{1}, x_{2}\right)>f_{2}\left(x_{1}, x_{1}\right)$ whenever $v_{2}\left(x_{1}\right)>\phi_{2}\left(x_{1}, x_{1}\right)$ 
The above example also shows that Corollary 2 is not implied by Theorem 1 of [2]. Assume further that $\phi_{1}(x) \geq 2$ and $\left|f_{2}(x)\right|,\left|\phi_{2}(x)\right| \leq 1$ for all $x \in[0,1]^{2}$. Let $x=(0, \varepsilon), y=(0,0)$ and $U(s, t)=\bar{u}_{1}(s, t)+\bar{u}_{2}(s, t)$ for all $s, t \in[0,1]^{2}$. Then, $U(x, y)=\phi_{1}(0,0)+f_{2}(0, \varepsilon)>\phi_{1}(0,0)+\phi_{2}(0,0)=U(y, y)$. However, for all $x^{\prime} \in X$ and $\delta>0$, there exists $z \in B_{\delta}(y)$ such that $U\left(x^{\prime}, z\right) \leq U(z, z)$. In fact, letting $z \in B_{\delta}(y)$ be such that $z_{2} \neq x_{1}^{\prime}$ and $z_{2}=z_{1}$, we obtain that $U(z, z)-U\left(x^{\prime}, z\right)=$ $\phi_{1}(z)+\phi_{2}(z)-u_{2}\left(z_{1}, x_{2}^{\prime}\right) \geq 0$ since $u_{2}\left(z_{1}, x_{2}^{\prime}\right)-\phi_{2}(z) \leq 2 \leq \phi_{1}(z)$. Thus, this game is not diagonally transfer continuous and so Theorem 1 of [2] does not apply.

Also, this example illustrates that Corollary 2 is more permissive than the corollary in [3]. In fact, if $f_{1}(x) \leq 0$ for all $x \in[0,1]^{2}$, then Corollary 2 implies that the above game has a Nash equilibrium for all functions $f_{1}$. In particular, this is so even when $f_{1}$ is not upper semicontinuous, a case in which their result cannot be applied. ${ }^{7}$

Proposition 3 below states that weak upper semicontinuity is indeed a weakening of upper semicontinuity. Together with Corollary 2, it implies that all upper semicontinuous and weakly payoff-secure games have a Nash equilibrium. Hence, Corollary 2 generalizes the corollary in [3].

Proposition 3 If $G$ is upper semicontinuous, then $G$ is weakly upper semicontinuous.

Proof. Let $(x, y, u) \in \Gamma$ and $i \in N$ be such that $u_{i}\left(x_{i}, y_{-i}\right) \neq u_{i}$. Let $\left\{\left(x_{k}, y_{k}\right)\right\}_{k=1}^{\infty}$ be such that $(x, y)=\lim _{k}\left(x_{k}, y_{k}\right)$ and $u=\lim _{k} \bar{u}\left(x_{k}, y_{k}\right)$. Then, for all $i \in N, u_{i}=$ $\overline{7 \text { Letting }} \phi_{2}=f_{2}$ and $f_{1}$ be upper semicontinuous, we obtain an example of a game that satisfies the assumptions of the corollary in [3] but not those of Theorem 3.1 in [5]. 
$\lim _{k} u_{i}\left(x_{i}^{k}, y_{-i}^{k}\right)$ and, since player $i$ 's payoff function is upper semicontinuous, we obtain that $u_{i}\left(x_{i}, y_{-i}\right) \geq u_{i}$. This inequality combined with $u_{i}\left(x_{i}, y_{-i}\right) \neq u_{i}$ implies that $u_{i}\left(x_{i}, y_{-i}\right)>u_{i}$. Hence, simply let $\hat{x}_{i}=x_{i}$.

Although Corollary 2 implies the corollary in Dasgupta and Maskin [3], the same does not hold regarding either Reny's or Baye, Tian and Zhou's existence results.

The following example shows that Corollary 2 does not imply Reny's existence theorem. Let $G$ be described by $N=\{1,2\}, X_{1}=X_{2}=[0,1], u_{1}: X \rightarrow \mathbb{R}$ be defined by

$$
u_{1}\left(x_{1}, x_{2}\right)=\left\{\begin{array}{l}
1 \text { if } x_{1} \leq 1 / 2, \\
0 \text { otherwise }
\end{array}\right.
$$

and $u_{2}: X \rightarrow \mathbb{R}$ be defined by $u_{2}\left(x_{1}, x_{2}\right)=1-u_{1}\left(x_{1}, x_{2}\right)$. It is clear that $G$ is compact, quasiconcave and reciprocally upper semicontinuous (the last property follows because $u_{1}+u_{2} \equiv 1$, hence continuous). The game $G$ is also payoff secure. This follows because $u_{2}$ is lower semicontinuous (see [5, Corollary 3.4]) and $u_{1}\left(0, x_{2}^{\prime}\right)>u_{1}(x)-\varepsilon$ for all $\varepsilon>0, x \in X$ and $x_{2}^{\prime} \in X_{2}$. Thus, we can apply Reny's theorem to this game. However, $G$ does not satisfy weak upper semicontinuity: Letting $x=y=(1 / 2,0)$ and $u=(1,1)$, we see that $(x, y, u) \in \Gamma$ (simply take $x_{k}=(1 / 2,0)$ and $y_{k}=(1 / 2(1+1 / k), 0)$ for all $k \in \mathbb{N})$, but for all $i \in N$ and all $\hat{x}_{i} \in X_{i}, u_{i}\left(\hat{x}_{i}, y_{-i}\right) \leq 1=u_{i}$.

Furthermore, the above example also shows that Corollary 2 does not imply Baye, Tian and Zhou's Theorem. Recall that this game is not weakly upper semicontinuous and so Corollary 2 does not apply. However, it is both diagonally transfer continuous 
and diagonally transfer quasiconcave, implying that Baye, Tian and Zhou's Theorem can be used to conclude that it has a Nash equilibrium. Let $(x, y) \in X \times X$ be such that $U(x, y)>U(y, y)$. Since $U(y, y)=u_{1}(y)+u_{2}(y)=1$ for all $y \in X$, it follows that $U(x, y)=2$ and $u_{1}\left(x_{1}, y_{2}\right)=u_{2}\left(y_{1}, x_{2}\right)=1$. Thus, $x_{1} \leq 1 / 2$ and $y_{1}>1 / 2$. Let $\delta>0$ be such that $\delta<y_{1}-1 / 2$ and $x^{\prime}=x$. Then, $U\left(x^{\prime}, z\right)=u_{1}\left(x_{1}, z_{2}\right)+u_{2}\left(z_{1}, x_{2}\right)=$ $2>1=U(z, z)$ for all $z \in B_{\delta}(y)$. Thus, the game is diagonally transfer continuous. Finally, we establish diagonal transfer quasiconcavity. Let $X^{m}=\left\{x^{1}, \ldots, x^{m}\right\}$ and define $y^{k}=(0,0)$ for all $1 \leq k \leq m$ and $Y^{m}=\left\{y^{1}, \ldots, y^{m}\right\}$. Then, for any subset $\left\{y^{k_{1}}, \ldots, y^{k_{s}}\right\}$ of $Y^{m}$, with $1 \leq s \leq m$, if $y^{k_{0}} \in \operatorname{co}\left(\left\{y^{k_{1}}, \ldots, y^{k_{s}}\right\}\right)$, then $y^{k_{0}}=(0,0)$ and so, for all $x^{k_{j}}, 1 \leq j \leq s, U\left(x^{k_{j}}, y^{k_{0}}\right)=u_{1}\left(x_{1}^{k_{j}}, 0\right)+u_{2}\left(0, x_{2}^{k_{j}}\right)=u_{1}\left(x_{1}^{k_{j}}, 0\right) \leq 1=$ $U\left(y^{k_{0}}, y^{k_{0}}\right)$. Hence, the game is diagonally transfer quasiconcave.

\subsection{Weakening Weak Upper Semicontinuity}

Corollary 2, together with [5, Theorem 3.1] and [1, Proposition 1], implies that there are two sets of sufficient conditions for the existence of Nash equilibria in compact, quasiconcave games. These conditions are easily comparable since our conditions strengthen the upper semicontinuity requirement while they weaken the lower semicontinuity one.

In this section we consider the effect of combining the weakest forms of both upper and lower semicontinuity, i.e., weak reciprocal upper semicontinuity and weak payoff security. Proposition 4 shows that these two conditions are enough to guarantee that 
the set of Nash equilibria is closed and that the limit points of a sequence of $\varepsilon-$ equilibria, with $\varepsilon$ converging to zero, are Nash equilibria. ${ }^{8}$

Proposition 4 Suppose that $G$ is compact, weakly reciprocal upper semicontinuous and weakly payoff secure. Then, the set of Nash equilibria is closed, and if $\left\{x_{k}\right\}_{k=1}^{\infty}$ is a converging sequence of $\varepsilon_{k}-$ equilibria, with $\left\{\varepsilon_{k}\right\}_{k=0}^{\infty} \subseteq \mathbb{R}_{+}$converging to zero, then $x=\lim _{k} x_{k}$ is a Nash equilibrium of $G$.

Proof. Note that the first statement follows from the second by taking $\varepsilon_{k}=0$ for all $k \in \mathbb{N}$. In order to establish the second statement, let $\left\{\varepsilon_{k}\right\}_{k=1}^{\infty} \subseteq \mathbb{R}_{+}$converge to zero, $\left\{x_{k}\right\}_{k=1}^{\infty} \subseteq X$ be a converging sequence such that $x_{k}$ is an $\varepsilon_{k}$ - equilibrium, for all $k \in$ $\mathbb{N}$, and let $x=\lim _{k} x_{k}$. Since $u$ is bounded, we may assume that $\left\{u\left(x_{k}\right)\right\}_{k=1}^{\infty}$ converges. Let $u=\lim _{k} u\left(x_{k}\right)$ and since $u_{i}\left(x_{k}\right)+\varepsilon_{k} \geq v_{i}\left(x_{-i}^{k}\right) \geq u_{i}\left(x_{k}\right)$ for all $i \in N$ and $k \in \mathbb{N}$, we obtain that $u=\lim _{k} v\left(x_{k}\right)$. By weak payoff security, $v_{i}$ is lower semicontinuous and so $v_{i}\left(x_{-i}\right) \leq u_{i}$ for all $i \in N$. If $(x, u) \in \operatorname{cl}(\operatorname{graph}(u)) \backslash \operatorname{graph}(u)$, then, by weak reciprocal upper semicontinuity, there exists $i \in N$ and $\hat{x}_{i} \in X_{i}$ such that $u_{i}\left(\hat{x}_{i}, x_{-i}\right)>u_{i}$ and so $v_{i}\left(x_{-i}\right) \geq u_{i}\left(\hat{x}_{i}, x_{-i}\right)>u_{i}$, a contradiction. Hence, $(x, u) \in \operatorname{graph}(u)$, implying that $v_{i}\left(x_{-i}\right) \leq u_{i}=u_{i}(x)$ for all $i \in N$. Hence, $u_{i}(x)=v_{i}\left(x_{-i}\right)$ for all $i \in N$ and so $x$ is a Nash equilibrium of $G$.

$\overline{8 \text { See }[5}$, Remark 3.1] for the case of better-reply secure games. When the game is reciprocally upper semicontinuous and weakly payoff secure, the conclusion that the set of Nash equilibria is closed follows also from Theorem 1 in R. Gatti "A Note on the Existence of Nash Equilibrium in Games with Discontinuous Payoffs, and an Application to First-Price Auctions", University of Cambridge, 2005. 
The following example shows that weak lower semicontinuity and the smoothness of the sum of players' payoff functions (and, hence, weak reciprocal upper semicontinuity) are not enough to guarantee the existence of a Nash equilibrium.

Let $G$ be a two-player game with $X_{1}=X_{2}=[0,1]$. Let $f: X \rightarrow \mathbb{R}$ be defined by $f(x)=1-\left(x_{1}-x_{2}\right)^{2}, A=\left\{x \in X: x_{1} \geq x_{2}\right\}, B=\left\{x \in X: x_{1}<x_{2}\right\}, C=\{(1,0)\}$ and, for every subset $D$ of $X, \chi_{D}$ denote the characteristic function of $D$. Define $u_{1}(x)=f(x) \chi_{A}(x)-\chi_{C}(x)$ and $u_{2}(x)=f(x) \chi_{B}(x)+\chi_{C}(x)$ for all $x \in X$. Thus,

$$
u_{1}(x)= \begin{cases}-1 & \text { if } x=(1,0) \\ 1-\left(x_{1}-x_{2}\right)^{2} & \text { if } x_{1} \geq x_{2} \text { and } x \neq(1,0) \\ 0 & \text { otherwise }\end{cases}
$$

and

$$
u_{2}(x)= \begin{cases}1 & \text { if } x=(1,0) \\ 1-\left(x_{1}-x_{2}\right)^{2} & \text { if } x_{1}<x_{2} \\ 0 & \text { otherwise. }\end{cases}
$$

Note that $G$ is compact and quasiconcave. Moreover, the sum of players' payoff functions, $u_{1}+u_{2}$, is equal to $f$ and therefore smooth. Furthermore, $v_{1}\left(x_{2}\right)=v_{2}\left(x_{1}\right)=1$ for all $x_{1}, x_{2} \in[0,1]$ and so $G$ is weakly payoff secure. However, $G$ has no Nash equilibrium. In fact, the graph of player 1's best-reply correspondence is the diagonal of $X$, whereas that of player 2 equals $C=\{(1,0)\}$, and so they do not intersect. 


\section{References}

[1] Bagh, A., And A. Jofre (2006): "Reciprocal Upper Semicontinuity and Better Reply Secure Games: A Comment," Econometrica, 74, 1715-1721.

[2] Baye, M., G. Tian, and J. Zhou (1993): "Characterizations of the Existence of Equilibria in Games with Discontinuous and Non-quasiconcave Payoffs," Rev. Econ. Stud., 60, 935-948.

[3] Dasgupta, P., And E. Maskin (1986): "The Existence of Equilibrium in Discontinuous Economic Games, I: Theory," Rev. Econ. Stud., 53, 1-26.

[4] Glicksberg, I. (1952): “A Further Generalization of the Kakutani Fixed Point Theorem, with Application to Nash Equilibrium Points," Proc. Amer. Math. Soc., $3,170-174$.

[5] Reny, P. (1999): "On the Existence of Pure and Mixed Strategy Equilibria in Discontinuous Games," Econometrica, 67, 1029-1056. 